\title{
Imbalanced expression of functional surface molecules in regulatory and effector T cells in systemic lupus erythematosus
}

\author{
D. Mesquita Júnior ${ }^{1 *}$, W.M. Cruvinel ${ }^{1,2 *}$, J.A.P. Araujo ${ }^{1}$, K.C. Salmazi ${ }^{3}$, \\ E.G. Kallas ${ }^{3}$ and L.E.C. Andrade ${ }^{1}$ \\ ${ }^{1}$ Disciplina de Reumatologia, Departamento de Medicina, Escola Paulista de Medicina, \\ Universidade Federal de São Paulo, São Paulo, SP, Brasil \\ ${ }^{2}$ Departamento de Biomedicina, Universidade Católica de Goiás, Goiânia, GO, Brasil \\ ${ }^{3}$ Disciplina de Imunologia Clínica e Alergia, Departamento de Clínica Médica, Faculdade de Medicina, \\ Universidade de São Paulo, São Paulo, SP, Brasil
}

\begin{abstract}
Regulatory T (TREG) cells play an important role in maintaining immune tolerance and avoiding autoimmunity. We analyzed the expression of membrane molecules in TREG and effector T cells in systemic lupus erythematosus (SLE). TREG and effector T cells were analyzed for the expression of CTLA-4, PD1, CD28, CD95, GITR, HLA-DR, OX40, CD40L, and CD45RO in 26 patients with active disease, 31 with inactive disease, and 26 healthy controls. TREG cells were defined as $\mathrm{CD} 25^{+/ \text {high }^{2}} \mathrm{CD} 127^{\varnothing / / \mathrm{low}} \mathrm{FoxP} 3^{+}$, and effector T cells were defined as CD25 ${ }^{+} \mathrm{CD} 127^{+} \mathrm{FoxP} 3^{\varnothing}$. The ratio of TREG to effector T cells expressing GITR, PD1, HLA-DR, OX40, CD40L, and CD45RO was determined in the three groups. The frequency of TREG cells was similar in patients with SLE and controls. However, SLE patients had a decreased frequency of CTLA-4 ${ }^{+}$TREG and CD28 ${ }^{+}$TREG cells and an increased frequency of CD40L ${ }^{+}$TREG cells. There was a decrease in the TREG/effector-T ratio for GITR ${ }^{+}, \mathrm{HLA}^{-\mathrm{DR}^{+}}, \mathrm{OX} \mathrm{O}^{+}$, and $\mathrm{CD} 45 \mathrm{RO}^{+}$cells, and an increased ratio of TREG/effector-T CD40L ${ }^{+}$cells in patients with SLE. In addition, CD40L ${ }^{+} \mathrm{TREG}$ cell frequency correlated with the SLE disease activity index $(P=0.0163)$. In conclusion, our findings showed several abnormalities in the expression of functionally critical surface molecules in TREG and effector T cells in SLE that may be relevant to the pathogenesis of this disease.
\end{abstract}

Key words: Systemic lupus erythematosus; T lymphocytes; Regulatory T cells; Effector T cells

\section{Introduction}

Understanding the pathophysiology of autoimmune diseases, and systemic lupus erythematosus (SLE) in particular, remains a great elusive challenge, but important advances have been achieved. With respect to cellular immunology, the characterization of regulatory $T$ (TREG) cells has had a considerable impact on the understanding of some aspects of several autoimmune diseases. TREG cells play an important role in maintaining immune tolerance (1). Their characteristic marker is the transcription factor FoxP3 (2), and an important advance in the study of TREG cells was the demonstration that low CD127 and high CD25 expression levels have a strong correlation with FoxP3 expression, therefore providing a valuable way to identify TREG cells based on membrane markers $(3,4)$. Profound deficiency in FoxP $3^{+}$TREG cells can potentially lead to the activation and expansion of rare self-reactive $T$ cells, thus inducing a wide spectrum of autoimmune/ inflammatory manifestations. In humans, this is exemplified by mutations in the FOXP3 gene that can result in the rare $X$-linked immunodeficiency syndrome known as IPEX (immune deregulation, polyendocrinopathy, enteropathy X-linked syndrome), a severe and rapidly fatal disorder with frequent autoimmune traits, such as thrombocytopenia and hemolytic anemia (5). It is not surprising that a number of TREG cell defects have been reported in patients with autoimmune diseases (6)

Studies in SLE patients have reported conflicting data regarding the frequency, phenotype, and function of these cells. The heterogeneity in findings of decreased (7-13), normal, or even increased frequency of TREG cells in

Correspondence: L.E.C. Andrade, Disciplina de Reumatologia, Departamento de Medicina, EPM, UNIFESP, 04023-900 São Paulo, SP, Brasil. Fax: +55-11-5576-4239. E-mail: luis.andrade@unifesp.br

${ }^{*}$ These authors contributed equally to this study.

Received August 29, 2013. Accepted June 9, 2014. First published online July 29, 2014. 
SLE (14-18) may be partially due to the criteria adopted in each study to define the TREG cell phenotype. We recently demonstrated that the $C D 25^{\text {high }}$ gate, considered by some studies as characteristic for TREG cells, contains high levels of activated effector $T$ cells in the active stages of SLE (4). Additional factors contributing to the heterogeneity of results include the clinical definition of disease activity, influence of immunosuppressant therapy, and technical aspects of in vitro cell handling, as well as flow cytometry analysis.

Several surface molecules are crucial for TREG cell survival and function. Some of these are constitutively expressed in TREG cells but are only expressed after activation in other $T$ cell subsets. One such molecule is cytotoxic T lymphocyte antigen 4 (CTLA-4), whose constitutive expression in TREG cells was shown to be under the control of FoxP3 (19). CTLA-4 plays an important role in contact-dependent suppression by means of the interaction between CTLA-4 on TREG cells with CD80/CD86 on effector $T$ cells, leading to downregulation of the latter. Alternatively, CTLA-4 on TREG cells can interact with CD80/CD86 on antigen-presenting cells and induce indoleamine dioxygenase expression in these cells. This enzyme depletes tryptophan and leads to the formation of toxic metabolites, resulting in local suppression of $\mathrm{T}$ cell proliferation (20). The closely related molecule PD-1 also acts to suppress $\mathrm{T}$ cell immune responses (20).

In addition, the expression of OX40, CD40L, and GITR (glucocorticoid-induced tumor necrosis factor receptor) at the effector $T$ cell surface could dictate the resistance of effector $T$ cells to TREG suppression. OX40 controls the development of effector and memory $T$ cells and is protective in the settings of cancer and infectious diseases but may be pathogenic in the setting of autoimmunity and allergy. As described by So et al. (21), OX40 displays dual activities supporting effector $\mathrm{T}$ cell development and concomitantly preventing the generation and activity of TREG cells. GITR is constitutively expressed by TREG cells but is also expressed by activated effector T cells. Previous studies have shown that the binding of GITR leads to antigen-nonspecific proliferation and activation of TREG cells, whereas the engagement of GITR renders effector T cells resistant to TREG suppression (22). These elements suggest that the balance of several surface molecules in TREG and effector $T$ cells may be relevant to the final fate of the interaction between these two $T$ cell subsets. In the present study, we investigated the balance of TREG and effector $T$ cells bearing the surface molecules CTLA-4, PD-1, OX40, GITR, CD40L, CD28, CD95, CD45RO, and HLA-DR in patients with active and inactive SLE.

\section{Material and Methods}

\section{Study population}

Patients were recruited from the Clinic at Universidade
Federal de São Paulo, São Paulo, SP, Brazil. All patients met the revised criteria of the American College of Rheumatology for SLE (23). Disease activity was rated by the SLE disease activity index (SLEDAI) (24) Active disease was defined as SLEDAI $\geqslant 6$, and inactive disease was $S L E D A I=0$. There were 26 patients with active disease $($ SLEDAI $=10.17 \pm 3.7)$ and 31 patients with no sign of disease activity (SLEDAI $=0)$. Twenty-six healthy volunteers (24 females and 2 males) were recruited from the hospital staff. All 26 patients with active SLE (A-SLE) were female; the 31 patients with inactive SLE (I-SLE) included 30 females and 1 male. There were no significant differences in the mean ages of healthy controls (29.81 \pm 8.2 years), A-SLE patients ( $36 \pm 11$ years), and I-SLE patients $(37 \pm 13$ years). The mean disease duration was $3.7 \pm 2.5$ years for patients with SLE. Most A-SLE patients were treated with monthly intravenous cyclophosphamide pulse therapy, and blood was drawn immediately before administration of this immunosuppressant. In addition, $15.4 \%$ of A-SLE patients used azathioprine, and $42.3 \%$ used hydroxychloroquine. In contrast, the treatment used by those with inactive disease included hydroxychloroquine (61.5\%), methotrexate $(7.7 \%)$ and low-dose prednisone (65.4\%). More detailed information about the clinical characteristics and clinical medications is provided in the Supplementary Table S1. Informed consent was obtained from all participants, and the study was approved by the UNIFESP Ethics Committee (CEP \#0037/06).

\section{Phenotypic evaluation of peripheral T lymphocytes}

Peripheral blood mononuclear cells (PBMC) were isolated by density gradient centrifugation in Ficoll Paque $^{T M}$ Plus (GE Healthcare Life Science, USA). PBMCs were washed in phosphate-buffered saline and $0.5 \times 10^{6}$ cells were incubated with fluorescein isothiocyanate (FITC) or allophycocyanin (APC)-labeled antiCD127, APC-Cy3-labeled anti-CD3, peridinin chlorophyll (PerCP)-labeled anti-CD4, and phycoerythrin (PE)-Cy7labeled anti-CD25 antibodies (Becton Dickinson, USA), according to the manufacturer's instructions. After $30 \mathrm{~min}$ of incubation at $4^{\circ} \mathrm{C}$, cells were washed with magnetic cell sorting (MACS) buffer, fixed and permeabilized with FoxP3 fixation/permeabilization buffer (eBioscience, USA), and then processed for FoxP3 staining using a kit containing APC-labeled anti-FoxP3 antibodies (eBioscience) according to the manufacturer's instructions. PE-labeled anti-CTLA-4, anti-PD1, anti-CD45RO, and anti-HLA-DR monoclonal antibodies and FITClabeled monoclonal antibodies to GITR, OX40, CD40L, CD28, and CD95 (Becton Dickinson) were used for phenotypic evaluation of TREG and effector $T$ cells following the same labeling protocol described above. Cells were processed using a FACSCanto flow cytometer (Becton Dickinson), and the acquired data were analyzed using the FlowJo software (Tree Star Inc., USA). 


\section{Statistical analysis}

Data are reported as means $\pm S D$. Statistical analysis was performed using one-way analysis of variance (ANOVA) for parametric variables and the KruskalWallis test for non-parametric variables. Differences were considered to be significant if $P$ values were 0.05 or less. Data analysis was performed using the Prism 5.0 software (GraphPad Software, USA)

\section{Results}

Frequency and phenotypic evaluation of peripheral blood CD25+/hiCD127-/lowFoxP3 + TREG cells in SLE

The gate strategy for TREG cell quantification was based on CD25 and CD127 expression, as shown in Figure 1. In the present study, we designated $\mathrm{CD}^{2} 5^{+} \mathrm{CD}_{12} 7^{\varnothing / \mathrm{llw}^{\circ}} \mathrm{FoxP}^{+}$cells as TREG-I cells and CD25 ${ }^{\text {high }}$ CD $127^{\varnothing / l^{\prime o w}} F o x P 3{ }^{+}$cells as TREG-II cells. The frequencies of these two TREG subsets were similar in A-SLE, I-SLE, and healthy controls (Figure 2).

In order to further characterize TREG cells based on the expression of regulatory, effector, and activation molecules, we determined the frequencies of TREG cells bearing CTLA-4, PD1, GITR, OX40, CD40L, CD28, CD95, CD45RO, and HLA-DR in both SLE patients and healthy controls. We observed that most TREG-I and

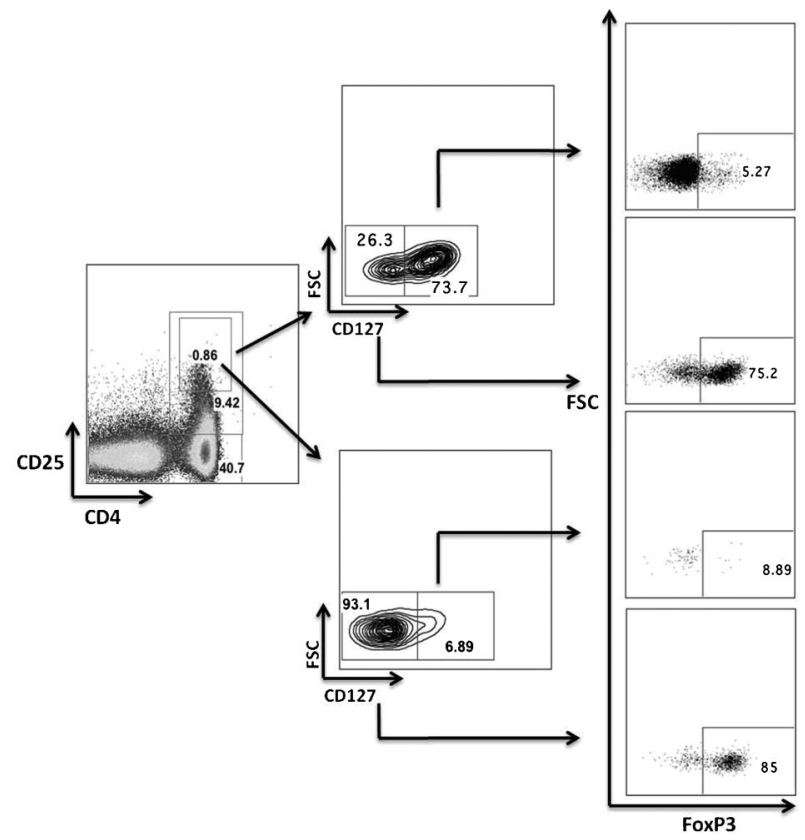

Figure 1. Multi-parameter strategy of analysis to identify TREG cells. A representative healthy control sample was analyzed by flow cytometry according to the expression of CD4, CD25, CD127, and FoxP3. CD25 ${ }^{+}$and $\mathrm{CD} 25^{\text {high }}$ cell subsets presented distinct frequency of CD127 $7^{\varnothing / \text { low }}$ cells and in both subsets the CD127 $7^{\varnothing / \text { low }}$ phenotype was associated with high FoxP3 expression.
TREG-II cells consistently expressed CD95, approximately half expressed CD45RO, and about one-third expressed HLA-DR, with no significant differences between SLE patients and healthy controls (Table 1). Almost all TREG cells were $\mathrm{CD}_{2}{ }^{+}$, and there was a decreased frequency of CD28 ${ }^{+}$TREG cells in the A-SLE group compared with healthy controls (Figure $3 A$ ). In addition there was an increased relative frequency of $\mathrm{CD} \mathrm{LL}^{+}$TREG-I and TREG-II cells in A-SLE patients compared with I-SLE and healthy controls (Figure $3 \mathrm{~B}$ ). The frequency of CTLA-4 ${ }^{+}$TREG-I cells was lower in ASLE patients than in healthy controls (Figure 3C). Disease activity, as assessed by SLEDAI, was weakly correlated with the frequency of CD40L ${ }^{+}$TREG-I cells (Figure 4) but not with the frequency of other TREG cell subsets (data not shown). There was an increased relative frequency of OX40 ${ }^{+}$TREG-I in I-SLE patients compared to A-SLE and healthy controls (Figure 5), but no differences were observed in the frequencies of $\mathrm{GITR}^{+}$TREG cells, OX $40^{+}$TREG cells, and PD $1^{+}$TREG cells between SLE patients and healthy controls (Figure 5). The representative flow cytometry dot plots of the evaluated molecules can be seen in Supplementary Figure S1.

\section{Altered ratio of TREG cells to effector T cells in SLE patients and healthy controls according to surface phenotype}

We determined the ratios of TREG to effector T cells bearing CD40L, CD28, GITR, OX40, PD1, CD45RO, HLA-DR, and CD95 surface molecules in both SLE patients and healthy controls. There were significantly lower TREG-I/effector T and TREG-II/effector T ratios for $\mathrm{CD} 4 \mathrm{RO}^{+}$cells in patients with A-SLE and I-SLE compared with healthy controls (Figure 6A). There was also a lower TREG-I/effector $\mathrm{T}$ ratio for $\mathrm{HLA}^{-\mathrm{DR}^{+}}$cells in A-SLE compared with I-SLE and healthy controls (Figure $6 \mathrm{~B})$. With respect to the effector molecules GITR and OX40, there were significantly lower TREG-I/effector T ratios for $\mathrm{GITR}^{+}$cells and for $\mathrm{OX} 40^{+}$cells in A-SLE patients compared with healthy controls (Figure $6 \mathrm{C}$ and D, respectively). Finally, there was a higher TREG-I and TREG-II/effector $\mathrm{T}$ ratio for $\mathrm{CD} 4 \mathrm{OL}^{+}$cells in A-SLE compared to I-SLE and healthy controls (Figure 6E). The TREG/effector $T$ ratio for PD1 ${ }^{+}$cells did not differ between the groups (Figure 6F).

\section{Discussion}

Modulation of the functional properties of TREG and effector $T$ cells is associated with variation in the expression of several membrane molecules (19-22). In the present study, we investigated the phenotypic profile of membrane molecules associated with functional activation and inhibition of TREG and effector T cells. Using a highly specific strategy for TREG cell identification based on the surface markers $\mathrm{CD} 25^{+} \mathrm{CD} 127^{\varnothing / \mathrm{low}}$ 


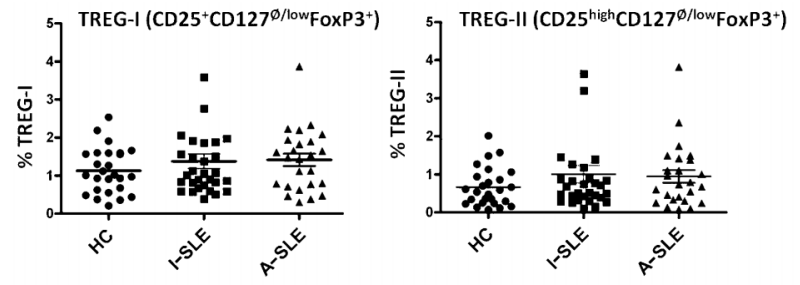

Figure 2. Patients with active SLE (A-SLE), inactive SLE (I-SLE), and healthy controls $(\mathrm{HC})$ display equivalent frequency of TREG cells. The relative frequency of TREG-I $\left(\mathrm{CD} 25^{+} \mathrm{CD} 127^{\text {Ø/low }} \mathrm{FoxP}^{+}{ }^{+}\right)$ and TREG-II $\left(\mathrm{CD} 25^{\text {high }} \mathrm{CD} 127^{\varnothing / \mathrm{low}^{\circ}} \mathrm{FoxP} 3^{+}\right)$cells was calculated over total $\mathrm{CD}^{+}$cells. Horizontal lines indicate means and standard deviation, respectively.

$(5-7,25)$, we could not find any abnormality in TREG cell frequency, but we originally identified a series of peculiar qualitative differences in the composition of surface molecules of TREG cells in SLE patients. Most TREG cells consistently expressed CD95, approximately half were $\mathrm{CD}_{45 \mathrm{RO}^{+}}$, and one-third were $\mathrm{HLA}^{-\mathrm{DR}^{+}}$in SLE patients and healthy controls. There was no difference between SLE and healthy controls regarding the frequencies of GITR $^{+}$TREG cells, OX40 ${ }^{+}$TREG cells, and PD1 ${ }^{+}$TREG cells. In contrast, samples from SLE patients exhibited lower frequencies of $\mathrm{CD}^{2} 8^{+}$TREG and $\mathrm{CTLA}-4^{+}$TREG cells compared with those from healthy controls. In addition there was an increased frequency of $\mathrm{CD} 40 \mathrm{~L}^{+}$TREG cells in samples from SLE patients, and the frequency of $\mathrm{CD} 4 \mathrm{~L}^{+}$TREG cells was correlated with disease activity.

The reduced frequency of CTLA-4 ${ }^{+}$TREG cells in ASLE is consistent with the previous demonstration of reduced CTLA-4 mRNA in $\mathrm{CD} 4{ }^{+} \mathrm{CD} 25^{+}$cells from SLE patients with active disease (12). The importance of CTLA-4 for TREG cell function was emphasized by Wing et al. (19), who showed that natural TREG cells may require CTLA-4 to suppress immune responses by affecting the ability of antigen-presenting cells to activate other T cells. CTLA-4 deficiency in TREG cells alone is sufficient to cause fatal autoimmune disease, whereas additional CTLA-4 deficiency in nonTREG cells improves the disease but was not sufficient to prevent autoimmunity in mice.

An accurate strategy for TREG cell identification by flow cytometry is critical to obtain meaningful results. CD127 expression has been shown to contribute to the distinction between effector T cells $\left(\mathrm{CD} 127^{+}\right)$and TREG cells $\left(C D 127^{\varnothing / l o w}\right)(5,6,25)$. Recently, we were able to show that this strategy is critical for appropriate TREG cell identification in patients with active SLE (7). We have shown that CD25 expression is a weak criterion for TREG cell identification in patients with active SLE in whom even the $T$ cell subset with highest CD25 expression contained a large proportion of FoxP3 ${ }^{\varnothing}$ cells (7).

Recent studies using well-defined TREG cell markers have not identified any major functional defect of TREG cells in human SLE; rather, they demonstrated abnormal resistance of effector $T$ cells to immune suppression by TREG cells $(13,17,18)$ and altered relative ratios of TREG versus effector $T$ cells in these patients (26). The present study contributes to this scenario by showing an increased frequency of TREG cells expressing CD40L in A-SLE patients compared with healthy controls and I-SLE patients, and a positive but weak correlation between the frequency of $\mathrm{CD} 4 \mathrm{~L}^{+} \mathrm{CD}^{+} 5^{+} \mathrm{CD} 127^{\varnothing / \mathrm{low}} \mathrm{FoxP} 3^{+}$and disease activity. The $\mathrm{CD} 25^{+} \mathrm{CD} 127^{\varnothing / \mathrm{low}} \mathrm{FoxP} 3^{+}$phenotype is exhibited not only by TREG cells but also by a significant fraction of activated effector $T$ cells, especially in SLE (7). In fact, increased CD40L expression and increased frequency of $\mathrm{CD} \mathrm{OL}^{+}$effector $\mathrm{T}$ cells have been investigated in association with SLE pathophysiology $(27,28)$. Blockade of the CD154/CD40 pathway was recently shown to reduce autoantibody production and to contribute to decreased disease activity $(29,30)$. These observations suggest that the increased frequency of $\mathrm{CD} 40 \mathrm{~L}^{+}$TREG cells reported here may be related to SLE disease activity.

Table 1. Relative frequency of TREG cells expressing HLA-DR, CD45RO, and CD95 in patients with SLE and HC.

\begin{tabular}{lcccc}
\hline Surface molecules & TREG cell subset & \multicolumn{3}{c}{ Group of individuals } \\
\cline { 3 - 5 } & & HC & I-SLE & A-SLE \\
\hline HLA-DR & TREG I & $22.60(12.25)$ & $26.33(13.84)$ & $25.59(12.22)$ \\
& TREG II & $35.55(14.31)$ & $33.06(19.43)$ & $29.15(17.51)$ \\
CD45RO & TREG I & $53.79(14.43)$ & $54.44(16.5)$ & $52.05(20.79)$ \\
& TREG II & $59.59(17.80)$ & $60.39(12.46)$ & $52.05(20.79)$ \\
CD95 & TREG I & $68.95(10.04)$ & $72.63(16.15)$ & $74.01(12.63)$ \\
& TREG II & $73.64(11.35)$ & $66.46(24.99)$ & $71.04(24.99)$ \\
\hline
\end{tabular}

HC: healthy control; I-SLE: inactive systemic lupus erythematosus (SLEDAI=0); A-SLE: active systemic lupus erythematosus

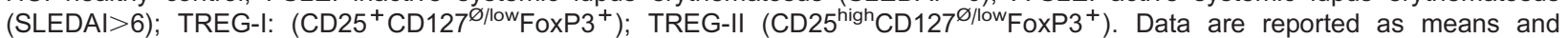
standard deviation of relative frequency of TREG I and TREG II positive for each molecule. There were no significant differences among groups (Kruskall-Wallis test). 

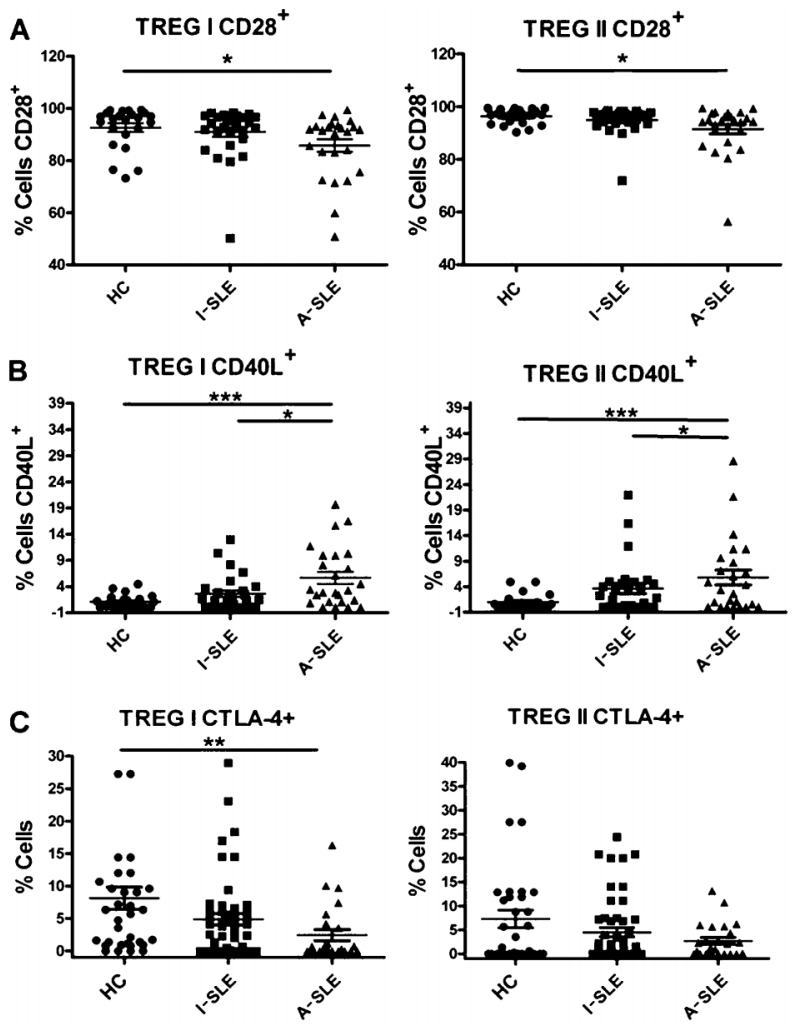

Figure 3. Analysis of the frequency of TREG-I (CD25 $5^{+}$ $\left.\mathrm{CD} 127^{\varnothing / \mathrm{low}} \mathrm{FoxP}^{+}\right)$and TREG-II (CD25 $\left.{ }^{\text {high }} \mathrm{CD} 127^{\text {Ø/low }} \mathrm{FoxP}^{+}\right)$ cells expressing CD28 $(A)$, CD40L $(B)$, and CTLA-4 $(C)$ in patients with active SLE (A-SLE), inactive SLE (I-SLE), and healthy controls $(\mathrm{HC})$. The relative frequency of $\mathrm{CD}_{2}{ }^{+}$, $\mathrm{CD} \mathrm{LL}^{+}$, and $\mathrm{CTLA}-4^{+}$cells was calculated over total TREG-I and TREG-II, respectively. Horizontal bars indicate means and standard deviation, respectively. ${ }^{*} \mathrm{P}<0.05$; ${ }^{* *} \mathrm{P}<0.01$; ${ }^{* * *} \mathrm{P}<0.001$ (Kruskal-Wallis test).

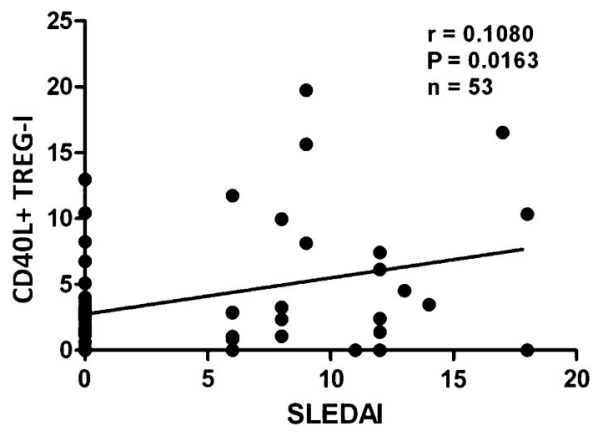

Figure 4. Correlation between the relative frequency of $\mathrm{CD} 40 \mathrm{~L}^{+}$ TREG-I cells and disease activity (SLEDAI) in patients with SLE. The relative frequency of $\mathrm{CD}_{40 \mathrm{~L}^{+}}{ }^{-}$TREG-I cells was calculated

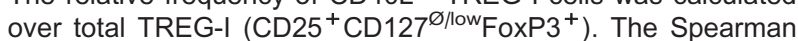
correlation test was used for analysis.

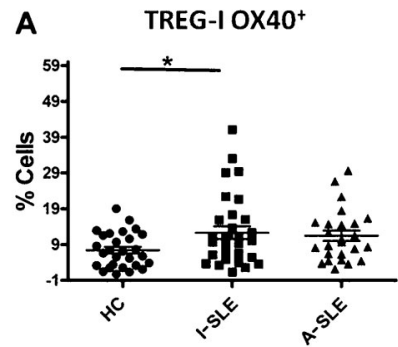

TREG-II OX40+

B

TREG-I PD1 ${ }^{+}$
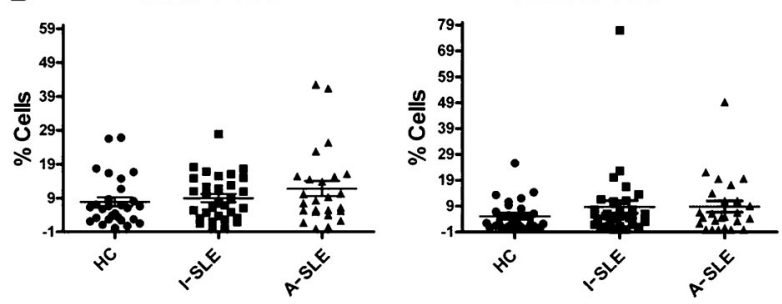

C

TREG-I GITR ${ }^{+}$
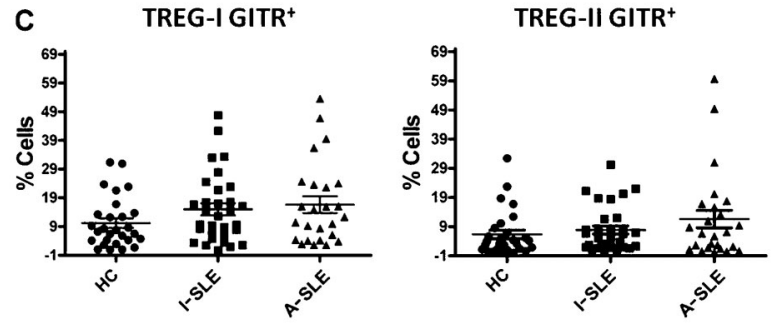

Figure 5. Analysis of the frequency of TREG-I $\left(C D 25^{+}\right.$

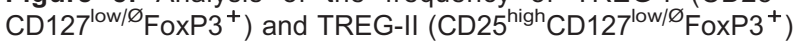
cells expressing OX40 $(A)$, PD1 $(B)$, and GITR $(C)$ in patients with active SLE (A-SLE), inactive SLE (I-SLE), and healthy controls $(\mathrm{HC})$. The relative frequency of $\mathrm{OX} 40^{+}, \mathrm{PD}^{+}$, and $\mathrm{GITR}^{+}$cells was calculated over total TREG-I and TREG-II, respectively. Horizontal bars indicate means and standard deviation, respectively. ${ }^{*} \mathrm{P}<0.05$ (Kruskal-Wallis test).

Patients with quiescent SLE displayed a higher frequency of OX40 ${ }^{+}$TREG cells compared with healthy controls. A recent study showed that OX40 costimulation did not significantly affect the proliferation or survival of naturally arising FoxP3 ${ }^{+}$TREG cells, but it profoundly inhibited FoxP3 gene expression (31), which can affect the transcriptional stability and functional behavior of TREG cells during the course of the disease. OX40, GITR, and CD40L are members of the TNF receptor super-family and play an important role in the immune response of effector and TREG cells. The expression of these molecules could make effector $T$ cells more pro-inflammatory and resistant to immune-regulation by TREG cells, whereas these molecules seem to be important for TREG cell proliferation and survival. Manipulation of these molecules in normal mice can lead to tolerance breakdown and induction of autoimmune disease similar to that produced by TREG cell depletion $(32,33)$. 

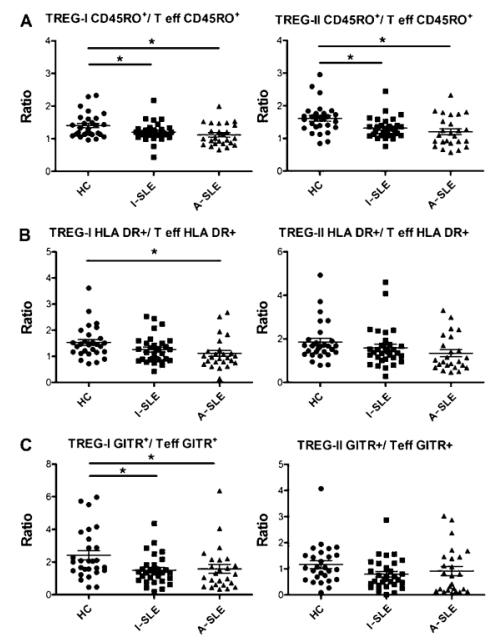
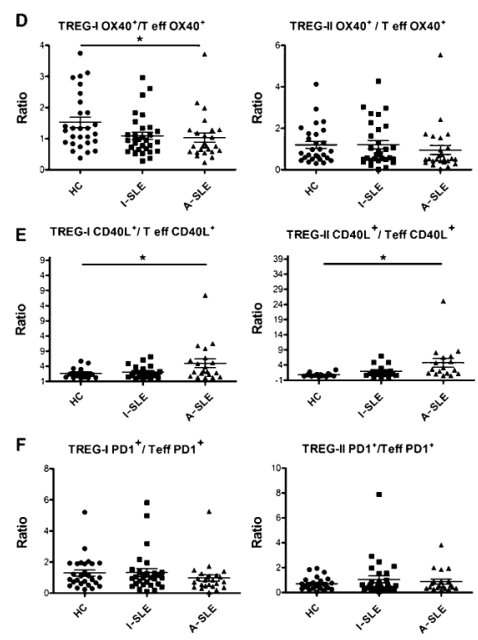

Figure 6. Ratio of TREG-I $\left(\mathrm{CD} 25^{+} \mathrm{CD} 127^{\varnothing / \mathrm{low}}\right.$ FoxP $\left.3^{+}\right)$or TREG-II (CD25 $5^{\text {high }}$ CD $127^{\varnothing / l o w ~}$ FoxP ${ }^{+}$) cells to effector $T$ cells expressing CD45RO $(A)$, HLA-DR $(B), \operatorname{GITR}(C)$, OX40 $(D)$, CD40L $(E)$, and PD1 $(F)$ in patients with active SLE (A-SLE), inactive SLE (I-SLE), and healthy controls $(\mathrm{HC})$. Horizontal bars indicate means and standard deviation, respectively. Teff: effector $\mathrm{T}$ cells. ${ }^{*} \mathrm{P}<0.05$ (Kruskall-Wallis test).
There was a lower frequency of CD28 ${ }^{+}$TREG cells in A-SLE compared to healthy controls, which indirectly points to higher levels of CD28 ${ }^{\text {null }}$ TREG cells in A-SLE. Loss of the CD28 molecule by CD4 cells is a hallmark of SLE. In fact, several studies have shown that the sustained stimulation of lymphocytes in chronic inflammatory processes may lead to loss of CD28 expression by $\mathrm{CD}^{+}$cells, which acquire alternate functions with pathogenic features and decreased susceptibility to immune regulation (34). Such CD4 + CD28 ${ }^{\text {null }}$ cells could be considered immune-senescent populations that may contribute to the development and exacerbation of autoimmunity. The CD28 molecule is also important in the generation of natural TREG cells, since naive CD28deficient CD4 T cells are deficient in upregulating FoxP3 in response to antigen and transforming growth factor beta (35). Therefore, the low frequency of CD28 ${ }^{+}$TREG cells may implicate decreased immune regulatory function in SLE patients with active disease.

In theory, immune responses are largely influenced by the final balance between TREG and effector $T$ cell function. Taking this point into consideration, we aimed to evaluate the ratio of TREG to effector T cells according to the expression of a collection of molecules that is biologically important for these cell subsets. We observed a gain in the memory subset $\mathrm{CD} 45 \mathrm{RO}^{+}$favoring effector $T$ cells over TREG cells in SLE patients. This finding is compatible with the high turnover of newly produced TREG cells (CD45RO ${ }^{\varnothing}$ TREG cells) and a relative decrease in the replacement of new naive effector $T$ cells, which was probably a consequence of limited immune space for newly generated cells, maintenance of activated effector cells, or decreased thymic output of novel T cells in SLE (36). Accordingly, we also showed a decreased ratio of TREG to effector T cells expressing the late activation marker HLA-DR and the effector molecules OX40 and GITR in A-SLE patients. This is the first demonstration of an imbalance favoring memory and activated TREG cells over effector T cells in SLE. A gain in memory and activated phenotypes for effector $T$ cells over TREG cells may be an important indicator of an unbalanced immune system in SLE that favors the effector proinflammatory pathway.

Several pieces of evidence indicate that deficiency or dysfunction of TREG cells produced by genetic abnormalities or environmental insults, even for a short period of time, could be sufficient to trigger autoimmunity in animal models $(35,37)$. The present demonstration of several qualitative alterations in TREG cell surface molecules in SLE patients might indicate functional impairment that could contribute to disease pathophysiology. The crosssectional nature of this study precludes any conclusion regarding a possible causal role for these abnormalities. Further studies are necessary to exclude the possibility that these abnormalities could be secondary to the immune disturbance specific to SLE or even to immunosuppressant therapy. This preliminary study did not include SLE patients with active disease and under no treatment due to the difficulty in finding those patients. Further studies are planned to extend this study to those rare patients.

In summary, the present study demonstrated that SLE patients with active disease exhibit an imbalance between TREG and effector $T$ cells in the expression of several phenotypic markers, including CTLA-4, CD40L, GITR, and OX40. These abnormalities may be relevant to the pathogenesis of SLE. In particular, the increased ratio of $\mathrm{CD} 4 \mathrm{~L}^{+}$TREG to $\mathrm{CD} 40 \mathrm{~L}^{+}$effector $\mathrm{T}$ cells in patients with active SLE is compatible with a scenario in which effector T cells would become more proinflammatory and resistant to immune regulation by TREG cells. Recently, major progress has been made in understanding the crucial role of TREG cells in the development of autoimmunity. The abnormalities reported here in the expression of functionally critical molecules by TREG and 
effector T cells may represent an additional piece to help solving the puzzle of SLE pathophysiology.

\section{Supplementary Material}

Click here to view [pdf].

\section{References}

1. Sakaguchi $\mathrm{S}$. Naturally arising $\mathrm{CD} 4+$ regulatory $t$ cells for immunologic self-tolerance and negative control of immune responses. Annu Rev Immunol 2004; 22: 531-562, doi: 10.1146/annurev.immunol.21.120601.141122.

2. Marson A, Kretschmer K, Frampton GM, Jacobsen ES, Polansky JK, Maclsaac KD, et al. Foxp3 occupancy and regulation of key target genes during T-cell stimulation. Nature 2007; 445: 931-935, doi: 10.1038/nature05478.

3. Bacchetta R, Passerini L, Gambineri E, Dai M, Allan SE, Perroni $\mathrm{L}$, et al. Defective regulatory and effector $\mathrm{T}$ cell functions in patients with FOXP3 mutations. $J$ Clin Invest 2006; 116: 1713-1722, doi: 10.1172/JCI25112.

4. Levings MK, Allan S, d'Hennezel E, Piccirillo CA. Functional dynamics of naturally occurring regulatory $T$ cells in health and autoimmunity. Adv Immunol 2006; 92: 119-155, doi: 10.1016/S0065-2776(06)92003-3.

5. Liu W, Putnam AL, Xu-Yu Z, Szot GL, Lee MR, Zhu S, et al. CD127 expression inversely correlates with FoxP3 and suppressive function of human CD4 $+\mathrm{T}$ reg cells. J Exp Med 2006; 203: 1701-1711, doi: 10.1084/jem.20060772.

6. Seddiki N, Santner-Nanan B, Martinson J, Zaunders J, Sasson S, Landay A, et al. Expression of interleukin (IL)-2 and IL-7 receptors discriminates between human regulatory and activated T cells. J Exp Med 2006; 203: 1693-1700, doi: 10.1084/jem.20060468.

7. Mesquita D, de Melo Cruvinel W, Araujo J, Pucci F, Salmazi $\mathrm{K}$, Kallas E, et al. Systemic lupus erythematosus exhibits a dynamic and continuum spectrum of effector/regulatory T cells. Scand J Rheumatol 2011; 40: 41-50, doi: 10.3109/ 03009742.2010.489229.

8. Crispin JC, Martinez A, Alocer-Varela J. Quantification of regulatory $T$ cells in patients with systemic lupus erythematosus. J Autoimmun 2003; 21: 273-276, doi: 10.1016/ S0896-8411(03)00121-5.

9. Liu MF, Wang CR, Fung LL, Wu CR. Decreased CD4 + CD25 + T cells in peripheral blood of patients with systemic lupus erythematosus. Scand J Immunol 2004; 59: 198-202, doi: 10.1111/j.0300-9475.2004.01370.x.

10. Miyara M, Amoura Z, Parizot C, Badoual C, Dorgham K, Trad S, et al. Global natural regulatory $T$ cell depletion in active systemic lupus erythematosus. J Immunol 2005; 175: 8392-8400, doi: 10.4049/jimmunol.175.12.8392.

11. Mellor-Pita S, Citores MJ, Castejon R, Tutor-Ureta $P$, Yebra-Bango M, Andreu JL, et al. Decrease of regulatory $\mathrm{T}$ cells in patients with systemic lupus erythematosus. Ann Rheum Dis 2006; 65: 553-554, doi: 10.1136/ard.2005. 044974.

12. Lee JH, Wang LC, Lin YT, Yang YH, Lin DT, Chiang BL. Inverse correlation between CD4 + regulatory T-cell population and autoantibody levels in paediatric patients with systemic lupus erythematosus. Immunology 2006; 117:

\section{Acknowledgments}

\author{
Research supported by FAPESP (\#2007/51349-2).
}

280-286, doi: 10.1111/j.1365-2567.2005.02306.x.

13. Valencia X, Yarboro C, Illei G, Lipsky PE. Deficient $\mathrm{CD} 4+\mathrm{CD} 25$ high $\mathrm{T}$ regulatory cell function in patients with active systemic lupus erythematosus. J Immunol 2007; 178: 2579-2588, doi: 10.4049/jimmunol.178.4.2579.

14. Suarez A, Lopez P, Gomez J, Gutierrez C. Enrichment of CD4 + CD25high T cell population in patients with systemic lupus erythematosus treated with glucocorticoids. Ann Rheum Dis 2006; 65: 1512-1517, doi: 10.1136/ard.2005. 049924.

15. Alvarado-Sanchez B, Hernandez-Castro B, Portales-Perez D, Baranda L, Layseca-Espinosa E, Abud-Mendoza C, et al. Regulatory $T$ cells in patients with systemic lupus erythematosus. J Autoimmun 2006; 27: 110-118, doi: 10.1016/ j.jaut.2006.06.005

16. Yates J, Whittington A, Mitchell P, Lechler RI, Lightstone L, Lombardi G. Natural regulatory T cells: number and function are normal in the majority of patients with lupus nephritis. Clin Exp Immunol 2008; 153: 44-55, doi: 10.1111/j.13652249.2008.03665.x.

17. Venigalla RK, Tretter T, Krienke S, Max R, Eckstein V, Blank N, et al. Reduced CD4 +, CD25- T cell sensitivity to the suppressive function of CD4 +, CD25high, CD127 -/low regulatory $T$ cells in patients with active systemic lupus erythematosus. Arthritis Rheum 2008; 58: 2120-2130, doi: 10.1002/art.23556.

18. Yan B, Ye S, Chen G, Kuang M, Shen N, Chen S Dysfunctional CD4 +, CD25 + regulatory T cells in untreated active systemic lupus erythematosus secondary to interferon-alpha-producing antigen-presenting cells. Arthritis Rheum 2008; 58: 801-812, doi: 10.1002/art.23268.

19. Wing $K$, Onishi $Y$, Prieto-Martin $P$, Yamaguchi $T$, Miyara M, Fehervari Z, et al. CTLA-4 control over Foxp3 + regulatory T cell function. Science 2008; 322: 271-275, doi: 10.1126/ science.1160062.

20. von Boehmer $\mathrm{H}$. Mechanisms of suppression by suppressor T cells. Nat Immunol 2005; 6: 338-344, doi: 10.1038/ni1180.

21. So T, Croft M. Cutting edge: OX40 inhibits TGF-beta- and antigen-driven conversion of naive CD4 T cells into CD25 + Foxp3 + T cells. J Immunol 2007; 179: 1427-1430, doi: 10.4049/jimmunol.179.3.1427.

22. Nocentini G, Riccardi C. GITR: a multifaceted regulator of immunity belonging to the tumor necrosis factor receptor superfamily. Eur J Immunol 2005; 35: 1016-1022, doi: 10.1002/eji.200425818.

23. Tan EM, Cohen AS, Fries JF, Masi AT, McShane DJ, Rothfield NF, et al. The 1982 revised criteria for the classification of systemic lupus erythematosus. Arthritis Rheum 1982; 25: 1271-1277, doi: 10.1002/art.1780251101.

24. Bombardier C, Gladman DD, Urowitz MB, Caron D, Chang $\mathrm{CH}$. Derivation of the SLEDAI. A disease activity index for 
lupus patients. The Committee on Prognosis Studies in SLE. Arthritis Rheum 1992; 35: 630-640, doi: 10.1002/art. 1780350606.

25. Hartigan-O'Connor DJ, Poon C, Sinclair E, McCune JM. Human CD4 + regulatory T cells express lower levels of the IL-7 receptor alpha chain (CD127), allowing consistent identification and sorting of live cells. J Immunol Methods 2007; 319: 41-52, doi: 10.1016/j.jim.2006.10.008.

26. Wan YY, Flavell RA. Regulatory T-cell functions are subverted and converted owing to attenuated Foxp3 expression. Nature 2007; 445: 766-770, doi: 10.1038/ nature05479.

27. Kim JM, Rasmussen JP, Rudensky AY. Regulatory T cells prevent catastrophic autoimmunity throughout the lifespan of mice. Nat Immunol 2007; 8: 191-197, doi: 10.1038/ ni1428.

28. Suen JL, Li HT, Jong YJ, Chiang BL, Yen JH. Altered homeostasis of $\mathrm{CD} 4(+)$ FoxP3(+) regulatory T-cell subpopulations in systemic lupus erythematosus. Immunology 2009; 127: 196-205, doi: 10.1111/j.1365-2567.2008. 02937.x.

29. Desai-Mehta A, Lu L, Ramsey-Goldman R, Datta SK. Hyperexpression of CD40 ligand by B and T cells in human lupus and its role in pathogenic autoantibody production. $J$ Clin Invest 1996; 97: 2063-2073, doi: 10.1172/JCI118643.

30. Devi BS, Van Noordin S, Krausz T, Davies KA. Peripheral blood lymphocytes in SLE - hyperexpression of CD154 on T and B lymphocytes and increased number of double negative T cells. J Autoimmun 1998; 11: 471-475, doi: 10.1006/jaut.1998.0213.

31. Boumpas DT, Furie R, Manzi S, Illei GG, Wallace DJ, Balow
JE, et al. A short course of BG9588 (anti-CD40 ligand antibody) improves serologic activity and decreases hematuria in patients with proliferative lupus glomerulonephritis. Arthritis Rheum 2003; 48: 719-727, doi: 10.1002/art.10856.

32. Grammer AC, Slota R, Fischer R, Gur H, Girschick H, Yarboro $\mathrm{C}$, et al. Abnormal germinal center reactions in systemic lupus erythematosus demonstrated by blockade of CD154-CD40 interactions. J Clin Invest 2003; 112: 15061520, doi: $10.1172 / \mathrm{JCl} 200319301$.

33. Vu MD, Xiao X, Gao W, Degauque N, Chen M, Kroemer A, et al. OX40 costimulation turns off Foxp3+ Tregs. Blood 2007; 110: 2501-2510, doi: 10.1182/blood-2007-01-070748.

34. Vijayakrishnan L, Slavik JM, Illes Z, Greenwald RJ, Rainbow D, Greve B, et al. An autoimmune diseaseassociated CTLA-4 splice variant lacking the B7 binding domain signals negatively in T cells. Immunity 2004; 20: 563-575, doi: 10.1016/S1074-7613(04)00110-4.

35. Kumanogoh A, Wang X, Lee I, Watanabe C, Kamanaka M, Shi W, et al. Increased T cell autoreactivity in the absence of CD40-CD40 ligand interactions: a role of CD40 in regulatory T cell development. J Immunol 2001; 166: 353-360, doi: 10.4049/jimmunol.166.1.353.

36. Thewissen $M$, Somers $V$, Hellings $N$, Fraussen $J$, Damoiseaux J, Stinissen P. CD4 +CD28null $T$ cells in autoimmune disease: pathogenic features and decreased susceptibility to immunoregulation. J Immunol 2007; 179 : 6514-6523, doi: 10.4049/jimmunol.179.10.6514.

37. Vieira QF, Kayser C, Kallas EG, Andrade LE. Decreased recent thymus emigrant number is associated with disease activity in systemic lupus erythematosus. I Rheumatol 2008; 35: 1762-1767. 